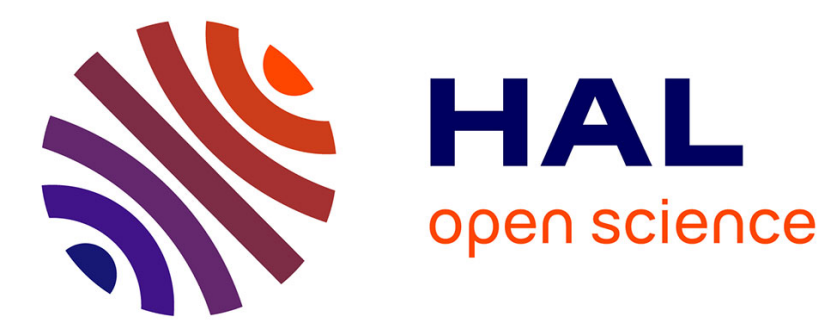

\title{
Détresse et douleurs sont-elles corrélées en fin de vie?
}

A. van Lander, J. Gaucher, Bruno Pereira, V. Virginie

\section{To cite this version:}

A. van Lander, J. Gaucher, Bruno Pereira, V. Virginie. Détresse et douleurs sont-elles corrélées en fin de vie?. Douleur et Analgésie, 2012, 25 (3), pp.175 - 182. 10.1007/s11724-012-0285-9 . hal-03119267

\section{HAL Id: hal-03119267 \\ https://hal.uca.fr/hal-03119267}

Submitted on 23 Jan 2021

HAL is a multi-disciplinary open access archive for the deposit and dissemination of scientific research documents, whether they are published or not. The documents may come from teaching and research institutions in France or abroad, or from public or private research centers.
L'archive ouverte pluridisciplinaire HAL, est destinée au dépôt et à la diffusion de documents scientifiques de niveau recherche, publiés ou non, émanant des établissements d'enseignement et de recherche français ou étrangers, des laboratoires publics ou privés. 


\section{Détresse et douleurs sont-elles corrélées en fin de vie ?}

\section{Pain and distress: are they correlated at the end of life?}

\section{A. Van Lander $\cdot$ J. Gaucher $\cdot$ B. Pereira $\cdot$ V. Virginie}

Reçu le 8 novembre 2011 ; accepté le 4 mai 2012

(C) Springer-Verlag France 2012

Résumé But : Cette recherche en psychologie étudie les liens entre effraction somatique, détresse et crise identitaire des patients en fin de vie.

Méthode : 14 psychologues analysent leurs entretiens à l'aide d'un livret réalisé à cet effet.

Résultats : 629 entretiens sont réalisés avec 210 patients. Leur détresse est fortement corrélée avec l'effraction de la fonction pare-excitatrice.

Conclusion : une effraction somatique augmente la détresse et rompt le sentiment d'unité. Cela se résorbe à proximité du décès.

Mots clés Soins palliatifs $\cdot$ Détresse $\cdot$ Crise d'identité Fonction pare-excitatrice $\cdot$ Psychologie

\begin{abstract}
Goal: This psychologic research studies the links between somatic break-in, distress and identity crisis of patients at the end of their life.

Method: 14 psychologists analyze their discussions with a specific booklet.

Results: 629 interviews are carried out with 210 patients. Their distress is strongly correlated with the breaking of the barrier-excitatory function.
\end{abstract}

\footnotetext{
A. Van Lander $(\bowtie)$

CHU Clermont-Ferrand, centre de soins palliatifs, hôpital Nord, F-63118 Cébazat

e-mail : avanlander@chu-clermontferrand.fr

Université de Lyon, laboratoire Santé, Individu, Société (EAM-SIS-HCL 4128)

J. Gaucher

Université de Lyon, laboratoire Santé, Individu,

Société (EAM-SIS-HCL 4128)

B. Pereira

CHU Clermont-Ferrand, DRCI

V. Virginie

CHU Clermont-Ferrand,

centre de soins palliatifs, centre de la douleur
}

Conclusion: a somatic breaking increases distress and breaks the sense of unity. It decreases near death.

Keywords Palliative care - Distress - Identity crisis · Barrier-excitatory function $\cdot$ Psychology

La détresse des hommes en fin de vie constitue le sujet de cette étude issue d'un travail de thèse doctorale de psychologie. Ces dix dernières années, ce sujet émerge dans l'ensemble des travaux menés par les équipes au contact des patients. La détresse est un défi pour les soins palliatifs [2]. Elle interpelle, convoque les familles et les soignants dans leurs limites à la supporter chez autrui. Elle devient l'enjeu de débats pour la société avec des réponses telles que l'euthanasie et le coma artificiel encore appelé sédation. L'insupportable de la détresse les justifie-t-elle ? Rend-elle la vie insensée ? La détresse en fin de vie est-elle une fatalité à laquelle nous ne pouvons répondre qu'en ôtant au patient sa conscience ou sa vie?

En cancérologie, aux États-Unis, à l'initiative de la National Comprehensive Cancer Network (NCCN), un groupe multidisciplinaire a proposé, à partir de 1997, des standards et des recommandations de bonnes pratiques cliniques pour l'évaluation et la prise en charge de la détresse $[7,8]$. La notion centrale est celle du retentissement du trouble psychique sur la vie de l'individu déterminant la nécessité d'aides. Holland et son équipe proposent une définition de la détresse, actuellement retenue en oncologie : «Une expérience désagréable de nature émotionnelle, psychologique ou spirituelle qui interfère avec l'aptitude à gérer son traitement. Elle se prolonge dans un continuum allant d'un sentiment commun normal de vulnérabilité, de tristesse, de peurs, jusqu'à des problématiques plus majeures comme une anxiété, des attaques de panique, une dépression, ou une crise spirituelle ». Celle-ci est considérée comme devant être entendue et traitée par l'équipe médicale intégrant une prise en charge psychosociale complémentaire. Parfois, ces réponses restent inefficaces face à des comportements de panique, d'agitations et des émotions extrêmes. Des équipes ont entrepris d'investiguer ses causes possibles au niveau social, 
spirituel et physiologique $[5,12,17,18]$ mais ses mécanismes psychologiques restent mal compris.

En soins palliatifs, elle représente selon Zittoun [20], « un défi pour la relation thérapeutique, plus encore que la mort ». Sa prévalence peut en effet atteindre jusqu'à $58 \%$ chez les patients en fin de vie [6]. Son lien avec le Mourir reste pourtant contradictoire. Si l'on en croit l'étude de Cheung et al. [1], qui utilisent l'Edmonton Symptom Assessment System (ESAS) pour évaluer 198 patients, la dépression et l'anxiété deviendraient même non significatives en se rapprochant du décès, mais cette équipe nuance ce résultat deux ans plus tard [19]. L'évaluation de 365 patients en ambulatoire à l'aide de la Beck Depression Inventory révèle en fait une augmentation de manière curviligne de la dépression. Il est à noter que la détresse continue à être évaluée le plus souvent à partir de la symptomatologie classique d'anxiété et de dépression avec de multiples échelles. Cette prolifération et dispersion des outils d'évaluation pourraient être le signe d'une difficulté à circonscrire une conception consensuelle. La définition proposée par Holland évitait la catégorisation psychiatrique du vécu du malade, particulier et « normal » d'une situation grave, mais elle relève d'avantage d'une proposition pragmatique. Avant d'expliciter plus avant cette nouvelle étude sur le vécu des patients en fin de vie, nous allons donc d'abord délimiter les concepts psychologiques complémentaires que nous retenons pour définir la détresse.

Pour commencer, l'étymologie du mot « détresse » renvoie à la même idée que celle de l'angoisse : être à l'étroit, oppressé avec l'idée d'une réduction des possibles. Le dictionnaire fondamental de psychologie (Bloch, 1997) propose cette définition : "Sentiment de délaissement, d'abandon, ressenti dans une situation critique, nécessitant une aide extérieure et/ou amenant un sujet à demander de l'aide, un secours social ou psychique (...). Le sujet en détresse souffre d'une disparition de ses investissements affectifs et d'une sidération de ses mécanismes de défense, pouvant le conduire, surtout dans la vieillesse, à une grave défaillance psychique (...) ». En psychanalyse, Freud utilisait le mot Hilflosigkeit, traduit par la notion de désaide, ce qui signifie être en manque d'aide. Ce terme désigne l'état d'extrême démunition, de vulnérabilité vitale de l'enfant dépendant dans ses premiers temps de vie de l'environnement maternel. Nous retiendrons donc de ce concept de détresse qu'il définit un état d'affect fort lié à la dépendance totale. Lorsque la dépendance est générée par une maladie grave, certains auteurs défendent en complément l'idée que cet affect puisse être l'expression d'une crise développementale d'identité [10]. La définition retenue pour la cancérologie par la NCNN évoque d'ailleurs la notion de " crise spirituelle». Appliquée à la période de la fin de la vie, cela voudrait dire que le Mourir constitue une étape de développement psychique, nécessaire pour intégrer dans l'identité en évolu- tion les changements imposés par la maladie. Le patient éprouve un sentiment de rupture de ses sentiments d'identité, dont le paroxysme est cette impression profonde de ne plus savoir qui il est : une perte de soi. Le vécu subjectif en serait la détresse. Dans la clinique, nous percevons effectivement cette dynamique identitaire associée à la détresse, en termes de déssaisissement de soi, mais également comme permettant parfois l'apparition de nouveaux sentiments d'intégrité [3].

Dans cette étude débutée en 2010, nous avons ainsi voulu observer à la fois la détresse et les mouvements identitaires chez les patients en fin de vie, dans l'objectif de repérer leur articulation avec l'évolution de la maladie. Parmi les nombreux résultats de ces observations, nous avons choisi, pour cet article, de sélectionner ceux qui nous permettent de répondre à ces questions : est-ce que la détresse psychique et les sentiments de ruptures identitaires sont liés aux pertes somatiques, notamment à la perte de la fonction pareexcitatrice responsable du vécu de douleur ? En d'autres termes, est-ce que la détresse est compréhensible au regard des fractures du corps ? Et enfin, est-elle sans issue ?

L'hypothèse que nous allons débattre est la suivante : les pertes somatiques, responsables notamment de la douleur, occasionnent des ruptures identitaires dont le vécu subjectif est la détresse.

\section{Méthode}

L'étude a été au préalable soumise au Comité d'Ethique des Centres d'Investigation Clinique de l'inter-région RhôneAlpes-Auvergne et au comité scientifique de la Société Française d'Accompagnement et de soins Palliatifs (SFAP). Elle est observationnelle et prospective sur une année : elle porte sur tous les entretiens cliniques réalisés par quatorze psychologues intervenant sur toutes les équipes de soins palliatifs (réseaux, équipes mobiles, lits identifiés et unité de soins palliatifs) de la région Auvergne, région présentant en France l'un des plus forts taux de mortalité (après le Limousin). Pour éviter les particularismes des interprétations de chaque psychologue, la méthodologie choisie est au carrefour entre méthode quantitative et qualitative. Le champ d'observation est constitué des entretiens : nous étudions les relations intersubjectives des psychologues et des patients, dans ces interactions où le troisième protagoniste est la maladie létale. L'interprétation compréhensive opérée par les psychologues dans les entretiens détecte les faits psychologiques pour nous aider à concevoir la rencontre psychique avec la maladie grave dans une sorte de schématisation générale des réalités individuelles. De façon très pratique, les psychologues ont signé un accord de collaboration, les engageant à utiliser durant une année un outil d'analyse. Ils se sont engagés à ne pas modifier leur pratique habituelle auprès de leurs patients et à utiliser l'outil a 
posteriori de chaque entretien dans la limite d'une semaine. Le fait d'utiliser les entretiens psychologiques réalisés en soins palliatifs comme champs d'observation a sélectionné les patients :

- présentant un niveau de vigilance suffisant pour être en capacité de bénéficier d'un entretien, c'est-à-dire coté un, deux et trois à l'échelle de Rudkin ;

- suivis par une équipe de soins palliatifs et atteints d'une pathologie grave, évolutive ou terminale ;

- en demande d'une aide psychologique qui soit à l'origine de la rencontre ;

- suivis potentiellement à domicile ou lors d'hospitalisation.

Pour aiguiser l'attention des psychologues, selon cette activité psychique décrite par Freud d'aller « à la rencontre des impressions des sens au lieu d'attendre passivement leur apparition » [4], l'outil introduit un système de marques facilitant la mémorisation et la restitution des éléments de l'entretien. Il s'agit d'une trame conceptuelle, sous forme d'un formulaire, à remplir pour évaluer : la détresse, la crise identitaire, l'importance des pertes générées par la pathologie, les mécanismes d'adaptation et de défense, les contretransferts des psychologues et les fonctions des entretiens. Chaque concept était expliqué aux psychologues dans un livret accompagnant le formulaire. Nous avons déjà présenté ailleurs les résultats concernant sa validité [13-15]. Les items qui nous intéressent ici sont ceux concernant les pertes, la crise identitaire, la détresse et les mécanismes de défense et d'adaptation.

Concernant tout d'abord les pertes, les psychologues avaient à leur disposition un tableau pour indiquer l'importance des pertes, notamment des fonctionnalités du corps, de l'image du corps et de la fonction pare-excitatrice. Ces trois catégories n'impliquent pas en fait les mêmes processus. Les pertes de l'image et des fonctionnalités du corps impliquent un mouvement de dessaisissement de soi, de réduction à la manière d'une peau de chagrin, alors que les pertes de la fonction pare-excitatrice impliquent un mouvement de multiplication envahissante de sensations douloureuses nouvelles. Examinons les pertes en termes de dessaisissement de soi :

- l'image du corps est l'ensemble des perceptions et des représentations qui nous servent à évoquer notre corps, comme objet physique mais aussi chargé d'affects. C'est l'aspect imageant du corps comme support affectif et comme première représentation inconsciente de soi, comme principe unificateur et médiateur de la relation à l'autre. Elle dépend de la qualité de l'environnement, ainsi que de la formation du narcissisme. À tout moment, elle peut être modifiée, à l'épreuve de la vie relationnelle ;

- les fonctionnalités du corps sont les fonctions de relations (qui permettent de se mouvoir, d'entendre, de s'exprimer, de toucher), les fonctions de nutrition (qui permettent de se maintenir en vie par les échanges avec notre environnement en s'alimentant, respirant, éliminant...) et les fonctions de reproduction. Lorsque des pertes se produisent au niveau des fonctionnalités du corps, par exemple des fonctions éliminatrices ou nutritives, elles réinterrogent la distinction entre soi et l'autre. La barrière imaginaire entre l'intérieur et l'extérieur n'est plus opérante. La limite du corps est trouée. Les pertes ne s'inscrivent pas simplement en termes de ce qui se détache ou sort du corps mais en termes de perte de soi.

Au contraire, de ces pertes de l'image et des fonctionnalités du corps, l'effraction de la fonction pare-excitatrice signifie pour les patients une augmentation des perceptions somatiques. La fonction pare-excitatrice permet de réguler les tensions internes ou externes au corps. Nous pouvons imaginer cette fonction ainsi qu'une peau, à la surface de notre corps, qui réduit et transforme ce qui peut nous atteindre. Développée à partir de l'enfance, elle permet de lier les perceptions avec des représentations pour les signifier et éviter qu'elles ne fassent effraction au niveau psychique. La tension est subjectivée et représentée. Nous supportons la douleur tant que nous la comprenons comme provenant d'un lieu circonscrit. Une perte de la fonction pare-excitatrice signifie une capacité moindre à supporter la douleur avec une origine que nous ne localisons plus en un lieu interne ou externe. D'enveloppe vide et trouée, le corps se remplit de nouvelles sensations. Elles envahissent le psychisme sidéré et effracté et réduisent la temporalité à ce présent figé.

Après l'évaluation de l'ensemble de ces pertes, les psychologues évaluaient dans le formulaire la fréquence de la détresse et du bien-être chez leurs patients au moyen d'une échelle visuelle analogique sur le modèle de celle élaborée par le groupe interdisciplinaire de Holland [7,8].

Concernant ensuite la crise identitaire, un tableau permettait d'indiquer si les patients présentaient une rupture ou une nouveauté des sentiments d'identité (d'unité, de continuité, d'originalité, de diversité, de soi réalisé par l'action et d'estime de soi). Ce tableau s'inspire des travaux d'Erikson [3], Kaës [9] et Tap [11]. Il a précédemment été utilisé dans une forme assez semblable par Welnovski-Michelet [16]. Nous allons expliciter les trois sentiments les plus impliqués dans le sujet de cet article : les sentiments d'unité, de continuité et de réalisation de soi par l'action. La première dimension développée par Erikson est la continuité, c'est-à-dire la permanence de soi dans le temps et l'espace. Malgré les différences identitaires, le sujet continue à s'éprouver le même. Il n'éprouve pas de rupture de son existence. Dans la maladie grave, les changements du corps peuvent rompre ce sentiment par des modifications trop rapides et nombreuses. Le second sentiment, le sentiment d'unité ou de cohérence à soi permet au sujet de se sentir comme « un » et non dilué avec autrui. Un effort constant d'autonomisation, de séparation et 
d'affirmation sont nécessaires pour que le sujet se sente en cohérence avec qui il est. Enfin, l'action est nécessaire à l'individu pour mettre en acte son potentiel. C'est le terreau des sentiments d'identité. L'action permet de s'affirmer au présent, mais également dans les différentes temporalités. Réaliser ses valeurs oriente sa vie, donne le sentiment d'en être acteur et procure une sensation de bien-être. On éprouve la vérité de ce que l'on est, de ce que l'on fait et de ce qu'on est capable de faire.

Concernant enfin les mécanismes de défense et d'adaptation, nous avons choisi d'associer des mécanismes pourtant très différents tels le Coping plutôt inspiré du courant de la psycho-oncologie, la dissociation plutôt psychanalytique et l'humour ou la rêverie mis en exergue par les travaux sur la résilience. Notre choix était guidé par notre expérience clinique et les études des thérapeutes intervenant auprès de patients en fin de vie comme Deschamps, Alric ou encore De M'Uzan. Nous avons ainsi proposé sept mécanismes conscients ou inconscients pouvant participer à la transformation de l'expérience traumatique : l'humour, la dissociation, la régression, les liens d'attachement, le Coping, l'intellectualisation et la rêverie. Ces mécanismes sont bien connus des soignants puisque classiquement évoqués pour comprendre les comportements des patients. Nous noterons simplement que la régression n'est pas à confondre avec des conduites de repli. Le patient investit son monde interne lorsque la fatigue devient intense. Même s'il paraît moins présent, il est toujours habité par ses pensées, le plaisir de se souvenir et d'imaginer. La présence bienveillante des soignants permet que cette régression soit confortable.

L'ensemble des paramètres étudiés au cours de cette étude a fait l'objet d'une analyse statistique effectuée par une équipe indépendante (DRCI-CHU Clermont-Ferrand) sous STATA v10 (Stata Corp, College Station, TX, ÉtatsUnis) et Alceste pour les notes libres des psychologues. La population a été décrite par des moyennes (M) avec les écarts-types $( \pm)$ et des médianes $(\mathrm{Md})$ pour les variables quantitatives et tous les tests statistiques ont été effectués au risque d'erreur de première espèce $\alpha=5 \%$ (hormis tests multiples). Nous indiquerons dans la suite de cet article les résultats significatifs en renseignant les coefficients de régression $[\mathrm{b}]$ accompagnés de leur intervalle de confiance codifié ainsi : (le premier intervalle/le second intervalle). Ils sont issus de modèles mixtes, adaptés pour l'analyse de données longitudinales puisque permettant, via l'introduction d'effets aléatoires, de modéliser la non-indépendance des données entre les différentes mesures d'un même individu au cours du temps. Les coefficients de régression indiquent le sens des relations entre deux paramètres (corrélation négative ou positive). Les ruptures identitaires sont indiquées par des valeurs négatives avec un score sur - 6 et les nouveautés identitaires avec des valeurs positives avec un score sur 6 .

\section{Résultats}

Les résultats sont issus de l'accompagnement de 210 patients et de 629 entretiens. Les patients étaient âgés en moyenne de 68,5 ans (33-95 ans) avec une majorité de femme (54,1\%). Leurs pathologies, principalement cancéreuses, dataient en moyenne de 23,8 mois $( \pm 41,4)$. Vingt-trois pourcents des patients étaient suivis à leur domicile. Ils étaient majoritairement informés de leur pathologie (98\%) et beaucoup du fait que leur pronostic était palliatif $(68,2)$. Leur décès est survenu peu après le premier entretien psychologique, une quarantaine de jours, ne laissant le temps, en général, que de trois entretiens.

Dans plus d'un entretien sur deux, les patients ont présenté des pertes générées par la maladie létale se rapprochant du niveau important. Les premiers entretiens ont présenté un niveau maximum de l'ensemble des pertes $(M=6,8 / 12)$. Les psychologues font référence à des moments de confusions et de grandes fatigues. Les pertes des fonctionnalités du corps sont en moyenne importantes $(2,2 / 3 \pm 0,8)$, les pertes de l'image du corps $(1,7 / 3 \pm 0,8)$ et celles de la fonction pareexcitatrice $(1,4 / 3 \pm 0,9)$ sont peu importantes. Les pertes des fonctionnalités et de l'image du corps sont très corrélées $[b=0,41]$. L'analyse longitudinale révèle une diminution de ces pertes en se rapprochant du décès (Fig. 1). Les psychologues ont évalué la détresse sur l'ensemble des entretiens à $3,9 / 10( \pm 1,9)$ avec une médiane à 4/10. Cinquante-trois pourcents des patients présentaient une détresse supérieure à $5 / 10$. L'analyse longitudinale révèle une fréquence maximale au premier entretien $(\mathrm{M}=4,7 / 10, \mathrm{Md}=5 / 10 \pm 2,8)$ atteignant le seuil identifié par la NCCN comme significatif (Fig. 2). Concernant les sentiments d'identité, les psychologues ont perçu des ruptures chez $77 \%$ des patients. La moitié des patients a présenté des ruptures d'au moins trois sentiments. Le sentiment de continuité était le plus atteint. Les psychologues ont perçu sa rupture dans $42,4 \%$ des entretiens. Le sentiment de soi réalisé par l'action était atteint

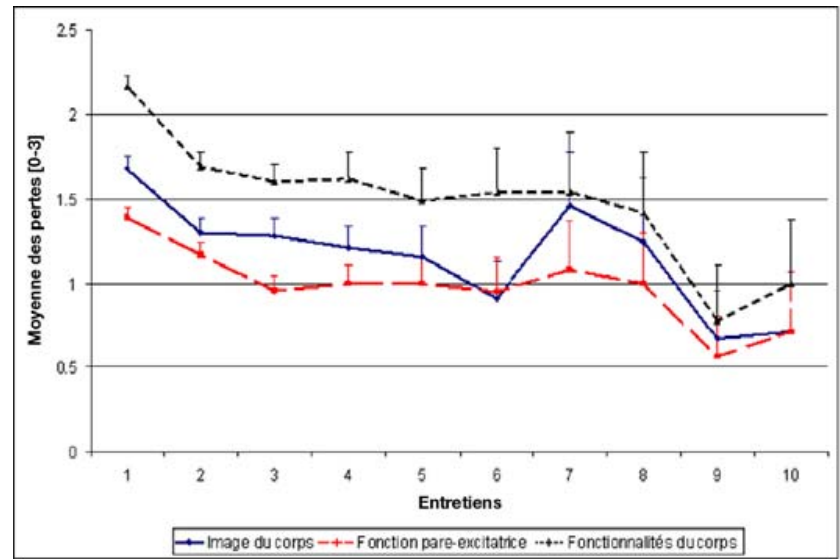

Fig. 1 Les pertes générées par la maladie létale $(\mathrm{n}=210)$ 


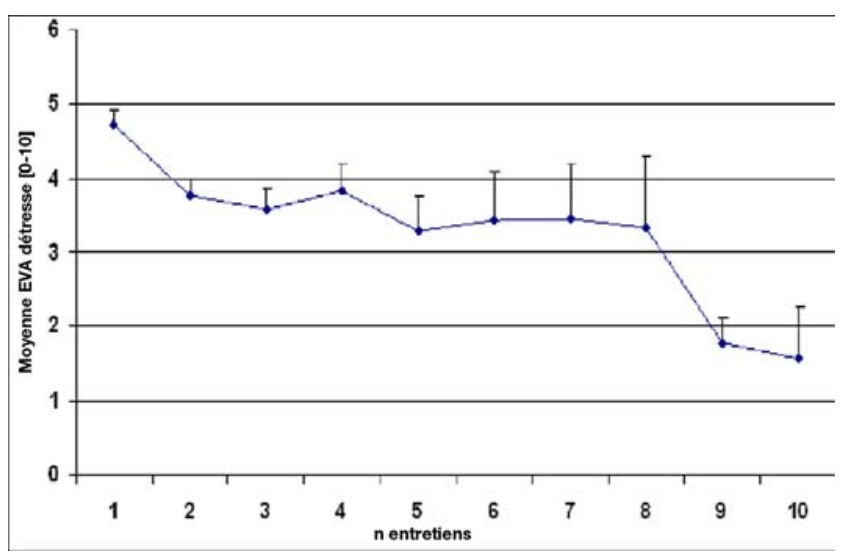

Fig. 2 La détresse ( $\mathrm{n}=210$ patients)

dans $34,9 \%$ des entretiens, le sentiment d'unité dans $29,2 \%$. Les autres sentiments étaient moins altérés. L'évolution longitudinale a été favorable. Les ruptures ont été en effet maximales lors du premier entretien avec une moyenne de $-2,3 /-6( \pm 1,8, \mathrm{Md}=-2)$ et régressaient ensuite (Fig. 3 ).

Concernant maintenant les corrélations entre les pertes somatiques et la crise identitaire, les résultats vérifient l'hypothèse selon laquelle les pertes occasionnent des ruptures identitaires. Lorsque les pertes sont évaluées comme régressant alors la crise identitaire se résorbe. Lorsqu'elles sont évaluées comme augmentant, la crise identitaire est plus présente $[b=-0,33(-0,39 /-0,27)]$. Les pertes de l'image du corps ont été les plus impliquées dans les crises identitaires $[b=-0,41$ $(-0,63 /-0,18)]$ avec une action sur les sentiments de continuité, d'unité et d'estime de soi. Les pertes des fonctionnalités du corps ont atteint le sentiment de soi réalisé par l'action $[b=-0,16(-0,21 /-0,09)]$. Les pertes de la fonction pare-excitatrice ont exercé un effet moindre $[b=-0,22(-0,43 /-0,006)]$ et uniquement sur le sentiment d'unité.

Ces ruptures identitaires sont ensuite très corrélées à l'apparition de la détresse $[b=0,7(0,32 / 1,08)]$ dans les entre-

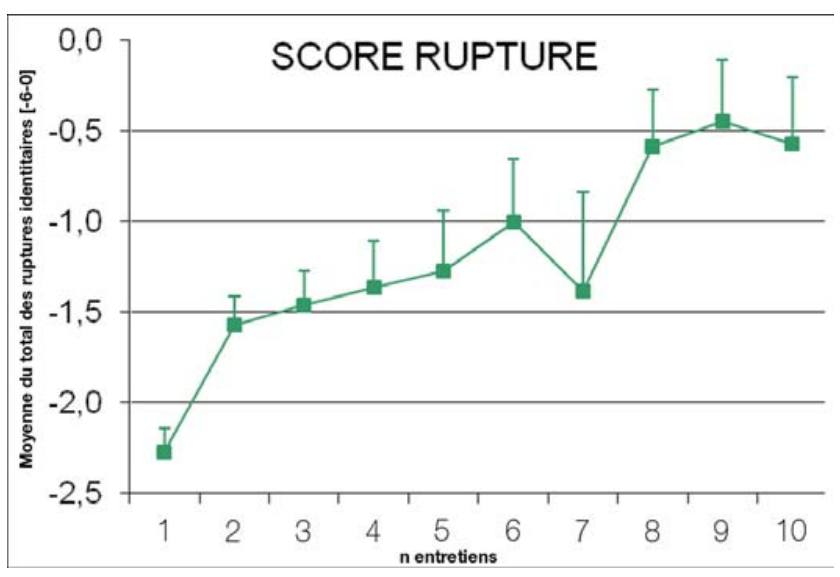

Fig. 3 Les ruptures identitaires $(n=210)$ tiens. En l'absence de rupture, une détresse significative n'était encore présente que dans seulement 6 à $11 \%$ des entretiens. Cette présence s'explique alors par les pertes de la fonction pare-excitatrice que nous avons vu peu impliquée dans la crise identitaire alors qu'elle générait directement une très forte détresse $[b=0,8(0,53 / 1,07)]$. Cela signifie que la présence de douleur forte en l'absence de crise identitaire peut générer une forte détresse. En retour, l'importance de la détresse augmentait également la sensation de perte chez les patients. Un patient avec une détresse élevée, c'est-à-dire supérieure à $4 / 10$, présentait un accroissement de ses pertes globales induites par la maladie $[\mathrm{b}=2(1,6 / 2,4)]$.

Concernant les mécanismes de défense, le mécanisme de régression était présent dans $50 \%$ des entretiens. Les pertes de la fonction pare-excitatrice et de l'image du corps augmentaient de façon importante chez les patients le recours à des comportements régressifs $[\mathrm{b}($ pare-excitatrice $)=0,65$ $(0,4 / 0,9)$ et $b($ image $)=0,35(0,11 / 0,57)]$. Les autres mécanismes de défense étaient plutôt mobilisés par la détresse et les ruptures identitaires. Les seuls mécanismes présentant pour effet de diminuer la détresse ont été la rêverie et 1 'humour $[\mathrm{b}$ (humour) $=-1,26(-2,03 /-0,49)$ et $b$ (rêverie $)=$ $-1,07(-1,9 /-0,25)]$. Peu présents en début de suivi, ils augmentaient ensuite progressivement. La rêverie permettait en outre de restaurer le sentiment de continuité $[b=0,28(0,07 / 0,49)]$ des patients ce qui, en retour, augmentait leur sentiment de bien-être $[\mathrm{b}=0,99(0,42 / 1,57)]$. Le mécanisme de dissociation était présent dans $54 \%$ des entretiens. Il n'était pas mobilisé par les pertes somatiques ni par la détresse mais par l'apparition de nouveaux sentiments d'identité $[b=0,59(0,43 / 0,75)]$ comme le sentiment de continuité que nous avons vu précédemment mobilisé par la rêverie. La dissociation a présenté cette caractéristique intéressante d'être activée par la dynamique identitaire qu'elle permettait également de potentialiser $[\mathrm{b}=0,3(0,07 / 0,53)]$.

En synthèse, les pertes de l'image et des fonctionnalités du corps généraient une crise identitaire au niveau des sentiments d'unité, de continuité et de réalisation de soi par l'action à l'origine d'une plus grande détresse alors que les pertes de la fonction pare-excitatrice n'impactent que le sentiment d'unité mais génèrent une très forte détresse. Les patients présentaient de plus fortes détresse et crise identitaire en début de suivi, ce qui s'accompagnait de comportements régressifs. Cela diminuait dans les entretiens suivants avec l'apparition de rêverie et d'humour.

\section{Discussion}

Dans ces résultats, nous allons essayer de comprendre l'effet respectif des pertes induites par la maladie sur l'identité et le 
vécu des patients en fin de vie. Nous rappelons que notre objectif était d'identifier si la détresse est compréhensible à la lumière des fractures du corps.

Parmi nos trois catégories, ce sont les pertes de la fonction pare-excitatrice qui occasionnent la plus forte détresse. Pour rappel cette fonction est celle qui est impliquée dans le vécu subjectif de la douleur. La revue de littérature confirme cette relation de cause à effet entre douleur et détresse. Déjà en 1920, Freud articulait sa théorie du traumatisme avec l'effraction du pare-excitation. Il précisait pourtant qu'une atteinte somatique protégeait l'individu du traumatisme psychique. L'énergie psychique, la libido se porte en effet sur la blessure. Elle est liée à une représentation et ne se transforme pas en angoisse envahissant le sujet. Le patient peut dire « j'ai mal à tel endroit» et non « je suis mal ». Les résultats de notre étude ne confirment pas ce rôle protecteur de la pathologie puisque les atteintes somatiques sont très corrélées avec la détresse psychique. Ceci provient certainement des caractéristiques des pathologies affectant les patients de notre étude : principalement cancéreuses avec des atteintes multiples de type métastatique. Ces pathologies ne sont pas localisées en un seul point, que peut se représenter le patient et sur lequel il peut focaliser son attention. La source somatique de douleur est multiple, diffuse et difficilement représentable. Au niveau identitaire, nous avons vu que la douleur exerce également un effet en termes de rupture sur le sentiment d'unité. Erikson évoque le sentiment d'unité en termes d'égalité à soi-même, d'unité de sa personnalité dont on soit fier. La douleur met à l'épreuve ce sentiment : le patient ne se reconnaît plus dans ses comportements honteux, d'appels désespérés et humiliants pour que la douleur cesse.

Les deux autres catégories de perte (image du corps et fonctionnalités) sont plutôt impliquées dans les ruptures identitaires qui occasionnent à leur tour la détresse. Les pertes des fonctionnalités du corps s'inscrivent du côté du manque, de la vulnérabilité et de la dépendance vitale à autrui. Elles réinterrogent la distinction entre soi et l'autre. Les fonctions éliminatrices sont par exemple assurées par les lavements, la pose de sonde... Les patients supportent progressivement qu'autrui ait accès à leur intimité avec une extériorisation permanente du corps pour le maîtriser. La maladie met à l'épreuve le sentiment d'unité. Elle transforme le corps en espace dont la géographie n'est plus familière. L'image du corps est atteinte. Ses éléments imaginaires sont détachés de leurs connexions habituelles pour devenir un assemblage d'organes défaillants dont le reflet est celui de l'étrangeté et du danger. Le sentiment de continuité est rompu avec cette évidence que plus rien n'est certain et que la permanence de soi n'est plus assurée. Les pertes des fonctionnalités, se conjuguant avec la perte d'une image unifiante du corps, déstabilisent la sensation de continuité et d'unité de soi alors que, dans le même temps, les moyens d'actions sont limités. Concernant le sentiment de soi réalisé par l'action, les tâches encore réalisables paraissent infantiles, se réduisant à répondre aux besoins primaires. Elles luttent difficilement contre le sentiment d'infériorité. La dépendance inscrit les sentiments d'insuffisance et d'incompétence à pourvoir à son existence par ses propres moyens.

L'ensemble des pertes qui accompagnent l'évolution de la maladie modifient le corps en termes de béance : perte de qualités et perte de composants, de «morceaux organiques ». Ces absences sont les signes apparents de la maladie qui se signifie dans le corps. Il est autre qu'avant et devient de plus en plus présent par les soins qu'il nécessite pour compenser ses pertes. Il devient le terrain visible du mauvais pronostic. Étymologiquement, le terme " maladie », " male habitus ", véhicule d'ailleurs l'idée de manière d'être, d'allure ou encore de façon générale l'idée de contenant, de vêtement. La maladie signifie une mauvaise façon d'habiter un corps de moins en moins ajusté à son propriétaire. Nous passons ainsi d'une dimension somatique à une dimension sémantique par l'impact au niveau identitaire. La maladie génère la détresse parce qu'elle fait sens au niveau de Soi. La crise du Mourir est celle de l'intégration d'un corps modifié et mortel avec cette question que l'on peut se poser, à la suite de Marin, " jusqu'où peut-on encore être soi-même » ?

Et en filigrane, comment évolue cette crise du mourir et quelle analgésie psychique est alors possible?

Étonnamment, nos résultats sont en contradiction avec notre expérience clinique et la logique médicale. En effet, selon les résultats de notre étude, les pertes des patients, leur détresse et les ruptures identitaires régressent avec l'avancée de la maladie jusqu'à présenter un niveau faible à proximité du décès.

La première explication réside certainement dans la prise en charge interdisciplinaire palliative. En effet, en complémentarité avec les psychologues, les médecins et les soignants interviennent pour limiter l'ensemble des symptômes notamment douloureux. Ces interventions conjointes ont certainement un effet thérapeutique sur les pertes de la fonction pare-excitatrice et ainsi sur la détresse qui en résulte.

La deuxième explication concerne l'interaction avec les mécanismes de défense. Le premier entretien est celui d'une prise de conscience du caractère définitif et irréductible des pertes. Le mécanisme de régression, très présent, peut être considéré comme une mobilisation par les patients de « contre-charges » venant stopper l'envahissement de la désorganisation, une forme de défense primaire destinée à juguler l'effraction et à dompter la poussée quantitative traumatique qui a effracté la fonction pare-excitatrice. Le patient se protège. Le corps douloureux redevient objet de soins et d'attentions. La maladie représente alors une pause, un recentrement sur soi. La durée de la détresse pourrait être considérée comme le temps nécessaire pour mobiliser les mécanismes plus évolués comme la dissociation, la rêverie et l'humour. Nos résultats concernant leur 
importance à proximité de la mort confirment les travaux de Rodin et Zimmermann [10]. Le patient présente une double conscience, deux attitudes psychiques différentes, opposées et indépendantes, l'une tournée vers la réalité et l'autre vers le désir. La dissociation permet l'apparition de nouveaux sentiments d'identité, notamment les sentiments de continuité. Pour mieux comprendre cette importance clinique de la dissociation et de la rêverie, nous allons évoquer ici un patient. Il a une cinquantaine d'année et vient d'apprendre le caractère mortel de son cancer. Atteint aux os, le risque important de fracture l'immobilise. Lors d'un entretien en présence de son épouse, il relate sa perception du caractère mortel de sa pathologie : aucune émotion ne transparait, il constate qu'il va mourir, certainement prochainement. Il est dans la réalité et en même temps au prix d'un éloignement de soi, figé dans un ressenti qui ne peut advenir. À l'entretien suivant, il explique au psychologue avoir très mal vécu les heures qui ont suivi l'entretien : une grande détresse qui l'a conduit à appeler très régulièrement l'équipe soignante. Il enchaîne rapidement sur son plaisir à imaginer, imaginer qu'il pourrait remarcher, se rappeler les moments agréables passés ou se projeter dans la venue prochaine de son épouse. Il conclut l'entretien sur ces mots : « j'ai préféré aujourd'hui parce qu'aujourd'hui, c'était agréable. C'est agréable de pouvoir espérer, même si je sais bien...». La dissociation, l'humour et la rêverie limitent l'impact psychique de l'effraction somatique.

Une dernière explication possible réside dans l'effet thérapeutique des entretiens. Les psychologues peuvent être considérés comme des objet-recours au sens de Roussillon ou Spitz pour soutenir les patients dans le travail de subjectivation de l'expérience traumatique. La mise en sens et la contenance des psychologues face à la crise identitaire permettent aux patients de se relier à soi et ainsi au corps modifié.

\section{Conclusion}

En conclusion, ces résultats concernant l'articulation entre effraction du corps, détresse et rupture des sentiments d'identité confirment l'hypothèse à l'origine de cette recherche : les pertes de la fonction pare-excitatrice mobilisent une plus grande détresse chez les patients et rompent le sentiment d'unité des patients. Les fractures du corps expliquent la détresse fréquente dans les situations de fin de vie en tant qu'elles génèrent une crise identitaire.

L'évolution longitudinale nous a par contre surpris. Aussi bien au niveau des pertes, de la détresse que de la crise identitaire, elle est favorable en se rapprochant du décès, qui pourtant normalement devrait être le temps de dépendance extrême. Les différentes pertes du corps régressent ce qui est corrélé à l'apparition d'état de bien- être et à ce que nous pourrions nommer une résilience identitaire. Qu'en comprendre?

La notion métaphorique de résilience identitaire peut permettre de rendre compte de cette reprise de dynamique : un rebond psychique en direction d'une possible métamorphose et élévation identitaire. À défaut de permettre aux patients de vivre leurs rêves, les entretiens psychologiques peuvent les aider à vivre une "identité résiliée », produit possible de cette psychodynamique retrouvée. La dissociation ou la rêverie contiennent la douleur, le corps est plus léger et ses atteintes moins prégnantes. Une issue à la détresse est possible. L'espoir ne réside pas dans le fait de ne pas mourir mais de pouvoir limiter l'impact psychique de l'effraction somatique pour se retrouver soi, suffisamment identique mais suffisamment transformé. Les résultats démontrent un mouvement identitaire possible malgré le dépérissement physique. Les patients évoluent d'un état de perte de soi à un mouvement de réconciliation avec un soi qui ne sera jamais achevé mais qui intègre la perte.

Ces questions sont importantes pour nous éclairer sur le fait que la détresse n'est pas une fatalité et n'impose pas des réponses irrémédiables. La relation thérapeutique prend tout son sens. Dans une autre forme d'approche, si on retient la notion de résilience, le thérapeute et les soignants ne seraientils pas en position de « tuteur de résilience »? C'est en tout cas la question forte qui s'impose à nous dans l'accompagnement des patients en fin de vie. Cette question à elle seule mériterait un travail de recherche plus approfondi.

Conflit d'intérêt : les auteurs déclarent ne pas avoir de conflit d'intérêt.

\section{Références}

1. Cheung WY, Barmala N, Zarinehbaf S, et al (2009) The association of physical and psychological symptom burden with time to death among palliative cancer out patients. J Pain Symptom Manage 37:297-304

2. Chochinov HM, Hassard T, McClement S, et al (2009) The landscape of distress in the terminally ill. J Pain Symptom Manage 38:641-9

3. Erikson E (1978) Adolescence et crise. La quête de l'identité. Flammarion, Manchecourt

4. Freud S (1984) Resultats, idées, problème. PUF, Paris, p 137

5. Gil F, Grassi L, Travado L, et al (2005) Use of distress and depression thermometers to measure psychosocial morbidity among southern european cancer patients. Support Care Cancer 13:600-6

6. Herbaud A, Reich M, Horner-Vallet D (2003) Évaluation de la détresse psychologique en soins palliatifs. À propos de 50 observations. InfoKara 18:5-10

7. Holland J (2003) Prise en charge de la détresse psychologique en cancérologie: standards et recommandation de bonnes pratiques cliniques. J Natl Compr Cancer Netw 1:344-74

8. Holland JC (1999) NCCN practice guidelines for the management of psycho-social distress. Oncology 5:113-47 
9. Kaës R (1979) Introduction à l'analyse transitionnelle. In: Crise, rupture et dépassement sous la direction de Kaës R. Dunod, Paris, pp 1-83

10. Rodin G, Zimmermann C (2008) Psychoanalytic reflections on mortality: a reconsideration. J Am Acad Psychoanal Dyn Psychiatry $36: 181-96$

11. Tap P (1998) Marquer sa différence. In: L'identité : l'individu, le groupe, la société sous la direction de Ruano Borbalan JC. Sciences Humaines, Auxerre, p 65

12. Tuinman MA, Gazendam-Donofrio SM, Hoekstra-Weebers JE (2008) Screening and referral for psychosocial distress in oncologic practice: use of the Distress Thermometer. Cancer 13:870-8

13. Van Lander A, Deveuve-Murol C, Pereira B, et al (2011) Entretiens psychologiques en soins palliatifs, caractéristiques des patients, spécificités des suivis. Rev Int Soins Pall 26:269-75

14. Van Lander A, Pereira B, Deveuve-Murol C (2010) Des psychologues en soins palliatifs réalisent un livret de méta-analyse. Perspective Psy 48:198-206
15. Van Lander A (2012) L'identité à l'épreuve de la maladie létale, un formulaire d'analyse qualitative des entretiens psychologiques. Psycho-Oncologie [sous presse]

16. Welnowski-Michelet $\mathrm{P}$ (2008) L'identité à l'épreuve de l'exclusion socioprofessionnelle. L'Harmattan, Paris

17. Zabora J, BrintzenhofeSzoc K, Curbow B, et al (2001) The prevalence of psychological distress by cancer site. PsychoOncology 10:19-28

18. Ziegler L, Hill K, Neilly L, et al (2011) Identifying psychological distress at key stages of the cancer illness trajectory: a systematic review of validated self-report measures. J Pain Symptom Manage 2:619-36

19. Zimmermann C, Cheung WY, Lo C, Rodin G (2011) Edmonton symptom Assessment System Screening and Depression at the End of Life. J Clin Oncol 29:3107-8

20. Zittoun R (2007) La mort de l'autre. Une introduction à l'éthique clinique. Dunod, Paris 